\title{
Iterative methods for the split common fixed point problem in Hilbert spaces
}

Huanhuan Cui and Fenghui Wang*

Dedicated to Professor Wataru Takashi on the occasion of his 70th birthday

*Correspondence:

wfenghui@gmail.com

Department of Mathematics,

Luoyang Normal University,

Luoyang, 471022, P.R. China

\begin{abstract}
The split common fixed point problem is an inverse problem that consists in finding an element in a fixed point set such that its image under a bounded linear operator belongs to another fixed point set. Recently Censor and Segal proposed an efficient algorithm for solving such a problem. However, to employ their algorithm, one needs to know prior information on the norm of the bounded linear operator. In this paper we propose a new algorithm that does not need any prior information of the operator norm, and we establish the weak convergence of the proposed algorithm under some mild assumptions.
\end{abstract}

MSC: 47J25; 47J20; 49N45; 65J15

Keywords: split common fixed point problem; directed operators; demicontractive operator; quasi-nonexpansive operator

\section{Introduction}

There has been growing interest in recent years in the split feasibility problem (SFP) [1]. The SFP is very useful in dealing with problems in signal processing and image reconstruction [2], especially in intensity-modulated radiation therapy [3]. Mathematically, the SFP is an inverse problem that consists in finding $\hat{x}$ with the property

$$
\hat{x} \in C, \quad \text { s.t. } \quad A \hat{x} \in Q \text {, }
$$

where $H$ and $K$ are two Hilbert spaces, $C$ and $Q$ are nonempty closed convex subsets of $H$ and $K$, respectively, and $A: H \rightarrow K$ is a bounded linear operator. In particular, if $C$ and $Q$ are composed of the fixed point sets of some nonlinear operators, then problem (1.1) is known as the split common fixed point problem (SCFP). More specifically, the SCFP consists in finding

$$
x \in \operatorname{Fix}(U), \quad \text { s.t. } \quad A x \in \operatorname{Fix}(T),
$$

where $\operatorname{Fix}(U)$ and $\operatorname{Fix}(T)$ stand for, respectively, the fixed point sets of $U: H \rightarrow H$ and $T: K \rightarrow K$.

\section{空 Springer}

(อ2014 Cui and Wang; licensee Springer. This is an Open Access article distributed under the terms of the Creative Commons Attribution License (http://creativecommons.org/licenses/by/2.0), which permits unrestricted use, distribution, and reproduction in any medium, provided the original work is properly cited. 
One method solving the SFP is Byrne's CQ algorithm [4], which generates a sequence $\left(x_{n}\right)$ by the recursive procedure:

$$
x_{n+1}=P_{C}\left(x_{n}-\rho_{n} A^{*}\left(I-P_{Q}\right) A x_{n}\right),
$$

where $\rho_{n}$ is known as the step, and $P_{C}, P_{Q}$ are the orthogonal projections onto $C$ and $Q$, respectively. When $C$ and $Q$ are simple in the sense that the associated projections are easily calculated, for example the half space, then the $C Q$ algorithm is efficient to solve the problem. However, if $C$ and $Q$ are complex sets, for example the fixed point sets, the efficiency of the $C Q$ algorithm will be affected because the projections onto such convex sets are generally hard to be accurately calculated. Alternatively, Censor and Segal [5] introduced the iterative scheme

$$
x_{n+1}=U\left(I-\rho_{n} A^{*}(I-T) A\right) x_{n}
$$

solving problem (1.2) for directed operators. Subsequently, this algorithm was extended to the case of quasi-nonexpansive [6] operators, demicontractive operators [7], and finitely many directed operators [8].

Let us now consider the SCFP (1.2) whenever $U$ and $T$ are directed operators. Then, if the step $\left(\rho_{n}\right)$ is chosen as

$$
0<\rho_{n} \equiv \rho<\frac{2}{\|A\|^{2}}
$$

algorithm (1.4) converges to a solution to problem (1.2) whenever such a solution exists. However, in order to implement this algorithm, one has first to compute (or, at least, estimate) the norm of $A$, which is in general not an easy work in practice. A natural question thus arises: Does there exist a way to select the step $\rho_{n}$ in algorithm (1.4) that does not depend on the operator norm $\|A\|$ ?

It is the purpose of this paper to answer the above question affirmatively. By introducing a new way of selections of the step, we obtain a method in a way that the implementation of algorithm (1.4) does not need any prior information of the operator norm. By using the Fejér monotonicity, we state the weak convergence of the new algorithm for demicontractive operators. Particular cases such as quasi-nonexpansive and directed operators are also considered.

\section{Preliminary and notation}

Throughout, let $I$ denote the identity operator, $\operatorname{Fix}(T)$ denote the set of the fixed points of an operator $T$, and let $\omega_{w}\left(x_{n}\right)$ denote the set of weak cluster points of the sequence $\left(x_{n}\right)$. The notation ' $\rightarrow$ ' stands for strong convergence and ' $\rightarrow$ ' stands for weak convergence. Given a nonempty closed convex subset $Q$ in $K$, let us define

$$
A^{-1}(Q):=\{x \in H: A x \in Q\}
$$

where $A: H \rightarrow K$ is a linear bounded operator. 
Definition 2.1 Assume $T: H \rightarrow H$ is a nonlinear operator. Then $I-T$ is saide to be demiclosed at zero, if, for any $\left(x_{n}\right)$ in $H$, the following implication holds:

$$
\left.\begin{array}{l}
x_{n} \rightarrow x \\
(I-T) x_{n} \rightarrow 0
\end{array}\right] \Rightarrow x \in \operatorname{Fix}(T) .
$$

It is well known that nonexpansive operators are demiclosed at zero (cf. [9]). Recall that an operator $T: H \rightarrow H$ is called nonexpansive if $\|T x-T y\| \leq\|x-y\|, \forall x, y \in H$.

Definition 2.2 Let $T: H \rightarrow H$ be an operator with $\operatorname{Fix}(T) \neq \emptyset$. Then

(i) $T: H \rightarrow H$ is called directed if

$$
\langle z-T x, x-T x\rangle \leq 0, \quad \forall z \in \operatorname{Fix}(T), x \in H
$$

(ii) $T: H \rightarrow H$ is called quasi-nonexpansive if

$$
\|T x-z\| \leq\|x-z\|, \quad \forall z \in \operatorname{Fix}(T), x \in H
$$

(iii) $T: H \rightarrow H$ is called $\tau$-demicontractive with $\tau<1$, if

$$
\|T x-z\|^{2} \leq\|x-z\|^{2}+\tau\|(I-T) x\|^{2}, \quad \forall z \in \operatorname{Fix}(T), x \in H,
$$

or equivalently

$$
\langle x-z, T x-x\rangle \leq \frac{\tau-1}{2}\|x-T x\|^{2}, \quad \forall z \in \operatorname{Fix}(T), x \in H .
$$

A typical example of a directed operator is an orthogonal projection $P_{C}$ from $H$ onto a nonempty closed convex subset $C \subset H$ defined by

$$
P_{C} x:=\underset{y \in C}{\arg \min }\|x-y\|^{2}, \quad x \in H .
$$

It is well known that the projection $P_{C}$ is characterized by

$$
P_{C} x \in C, \quad\left\langle x-P_{C} x, z-P_{C} x\right\rangle \leq 0, \quad \forall z \in C .
$$

Given a sequence $\left(x_{n}\right)$ in $H$, then $\left(x_{n}\right)$ is called Fejér monotone with respect to $C$, if

$$
\left\|x_{n+1}-c\right\| \leq\left\|x_{n}-c\right\|, \quad \forall n, \forall c \in C
$$

The sequence with Fejér monotonicity has the following property.

Lemma 2.3 [10] If the sequence $\left(x_{n}\right)$ is Féjer monotone w.r.t. a nonempty closed convex subset $C$, then $\left\{P_{C} x_{n}\right\}$ converges strongly; moreover,

$$
x_{n} \rightarrow x^{*} \in C \quad \Leftrightarrow \quad \omega_{w}\left(x_{n}\right) \subset C .
$$


Lemma 2.4 If $U: H \rightarrow H$ is $\kappa$-demicontractive, then

$$
\left\|U_{\lambda} x-z\right\|^{2} \leq\|x-z\|^{2}-\lambda(1-\kappa-\lambda)\|(I-U) x\|^{2},
$$

where $x \in H, z \in \operatorname{Fix}(U)$, and $U_{\lambda}:=(1-\lambda) I+\lambda U(0<\lambda<1-\kappa)$.

Proof First we deduce that

$$
\begin{aligned}
\left\|U_{\lambda} x-z\right\|^{2} & =\|(x-z)+\lambda(U x-x)\|^{2} \\
& =\|x-z\|^{2}+2 \lambda\langle U x-x, x-z\rangle+\lambda^{2}\|U x-x\|^{2},
\end{aligned}
$$

and using inequality (2.1) we have

$$
2 \lambda\langle U x-x, x-z\rangle \leq \lambda(\kappa-1)\|U x-x\|^{2} .
$$

Adding up these two formulas yields

$$
\left\|U_{\lambda} x-z\right\|^{2} \leq\|x-z\|^{2}-\lambda(1-\kappa-\lambda)\|U x-x\|^{2},
$$

which is the inequality as desired.

Lemma 2.5 Let $A: H \rightarrow K$ be a bounded linear operator and $T: K \rightarrow K$ a $\tau$-demicontractive operator with $\tau<1$. If $A^{-1}(\operatorname{Fix}(T))$ is nonempty, then

$$
(I-T) A x=0 \quad \Leftrightarrow \quad A^{*}(I-T) A x=0, \quad \forall x \in H .
$$

Proof It is clear that $(I-T) A x=0 \Rightarrow A^{*}(I-T) A x=0, x \in H$. To see the converse, let $x \in H$ such that $A^{*}(I-T) A x=0$. Taking $z \in A^{-1}(\operatorname{Fix}(T))$,

$$
\begin{aligned}
\frac{1-\tau}{2}\|(I-T) A x\|^{2} & \leq\langle(I-T) A x, A x-A z\rangle \\
& =\left\langle A^{*}(I-T) A x, x-z\right\rangle=0,
\end{aligned}
$$

where the inequality follows from (2.1), so that $A x=T(A x)$. Hence the proof is complete.

Lemma 2.6 Let $A: H \rightarrow K$ be a bounded linear operator and $T: K \rightarrow K$ a $\tau$-demicontractive operator with $\tau<1$. If $A^{-1}(\operatorname{Fix}(T)) \neq \emptyset$, then

$$
\left\|x-\rho A^{*}(I-T) A x-z\right\|^{2} \leq\|x-z\|^{2}-\frac{(1-\tau)^{2}}{4} \frac{\|(I-T) A x\|^{4}}{\left\|A^{*}(I-T) A x\right\|^{2}},
$$

where $x \in H, A x \neq T(A x), z \in A^{-1}(\operatorname{Fix}(T))$ and

$$
\rho:=\frac{(1-\tau)\|(I-T) A x\|^{2}}{2\left\|A^{*}(I-T) A x\right\|^{2}} .
$$


Proof Take $x \in H, A x \neq T(A x)$. Then from the previous lemma $\rho$ is well defined. Since $T$ is $\tau$-demicontractive,

$$
\begin{aligned}
\left\|x-\rho A^{*}(I-T) A x-z\right\|^{2} & =\|x-z\|^{2}-2 \rho\left\langle A^{*}(I-T) A x, x-z\right\rangle+\rho^{2}\left\|A^{*}(I-T) A x\right\|^{2} \\
& =\|x-z\|^{2}-2 \rho((I-T) A x, A x-A z\rangle+\rho^{2}\left\|A^{*}(I-T) A x\right\|^{2} \\
& \leq\|x-z\|^{2}-(1-\tau) \rho\|(I-T) A x\|^{2}+\rho^{2}\left\|A^{*}(I-T) A x\right\|^{2} \\
& =\|x-z\|^{2}-\frac{(1-\tau)^{2}}{4} \frac{\|(I-T) A x\|^{4}}{\left\|A^{*}(I-T) A x\right\|^{2}},
\end{aligned}
$$

where the inequality follows from (2.1).

\section{A new iterative algorithm}

Let us first consider the SCFP (1.2) for demicontractive operators. More specifically, we make use of the following assumptions:

- $U: H \rightarrow H$ is $\kappa$-demicontractive with $\kappa<1$;

- $T: K \rightarrow K$ is $\tau$-demicontractive with $\tau<1$;

- both $I-U$ and $I-T$ are demiclosed at zero;

- it is consistent, i.e., its solution set, denoted by $S$, is nonempty.

Under these conditions, we propose the following algorithm.

Algorithm 3.1 Choose $0<\lambda<1-\tau$ and an initial guess $x_{0} \in H$ arbitrarily. Assume that the $n$th iterate $x_{n}$ has been constructed; then calculate the $(n+1)$ th iterate $x_{n+1}$ via the formula:

$$
x_{n+1}=U_{\lambda}\left(x_{n}-\rho_{n} A^{*}(I-T) A x_{n}\right),
$$

where the step $\rho_{n}$ is chosen in such a way that

$$
\rho_{n}:= \begin{cases}\frac{(1-\tau)\left\|(I-T) A x_{n}\right\|^{2}}{2\left\|A^{*}(I-T) A x_{n}\right\|^{2}}, & A x_{n} \neq T\left(A x_{n}\right) ; \\ 0, & \text { otherwise. }\end{cases}
$$

Remark 3.2 By Lemma 2.5, we see that $A x_{n}=T\left(A x_{n}\right)$ if and only if $A^{*}(I-T) A x_{n}=0$. So Algorithm 3.1 is well defined.

Theorem 3.3 Let $\left(x_{n}\right)$ be the sequence generated by Algorithm 3.1. Then $\left(x_{n}\right)$ converges weakly to a solution $x^{*} \in S$.

Proof First we verify that $\left(x_{n}\right)$ is Féjer-monotone w.r.t. $S$. To see this, let $y_{n}:=x_{n}-\rho_{n} A^{*}(I-$ $T) A x_{n}$ and fix $z \in S$. For the case $\rho_{n}=0$, we have $y_{n}=x_{n}$ and by (2.4)

$$
\left\|x_{n+1}-z\right\|^{2}=\left\|U_{\lambda} x_{n}-z\right\|^{2} \leq\left\|x_{n}-z\right\|^{2}-\lambda(1-\kappa-\lambda)\left\|(I-U) x_{n}\right\|^{2},
$$

which implies $\left\|x_{n+1}-z\right\| \leq\left\|x_{n}-z\right\|$ because $0<\lambda<1-\kappa$. For the case $\rho_{n} \neq 0$, we deduce from (2.4)-(2.5) that

$$
\begin{aligned}
\left\|x_{n+1}-z\right\|^{2} & =\left\|U_{\lambda} y_{n}-z\right\|^{2} \\
& \leq\left\|y_{n}-z\right\|^{2}-\lambda(1-\kappa-\lambda)\left\|(I-U) y_{n}\right\|^{2}
\end{aligned}
$$




$$
\begin{aligned}
& =\left\|x_{n}-\rho_{n} A^{*}(I-T) A x_{n}-z\right\|^{2}-\lambda(1-\kappa-\lambda)\left\|(I-U) y_{n}\right\|^{2} \\
& \leq\left\|x_{n}-z\right\|^{2}-\frac{(1-\tau)^{2}}{4} \frac{\left\|(I-T) A x_{n}\right\|^{4}}{\left\|A^{*}(I-T) A x_{n}\right\|^{2}} \lambda(1-\kappa-\lambda)\left\|(I-U) y_{n}\right\|^{2} .
\end{aligned}
$$

Hence we have shown in both cases that $\left\|x_{n+1}-z\right\| \leq\left\|x_{n}-z\right\|$. Consequently $\left(x_{n}\right)$ is Féjermonotone w.r.t. $S$ and $\left(\left\|x_{n}-z\right\|^{2}\right)$ is therefore a convergent sequence.

We next show the following facts:

$$
\left\|(I-T) A x_{n}\right\| \rightarrow 0, \quad\left\|(I-U) x_{n}\right\| \rightarrow 0, \quad \text { as } n \rightarrow \infty .
$$

If $\rho_{n}=0$, it is clear that $(I-T) A x_{n}=0$, and in view of (3.1)

$$
\lambda(1-\kappa-\lambda)\left\|(I-U) x_{n}\right\|^{2} \leq\left(\left\|x_{n}-z\right\|^{2}-\left\|x_{n+1}-z\right\|^{2}\right) \rightarrow 0,
$$

because $\left(\left\|x_{n}-z\right\|^{2}\right)$ is convergent. Otherwise, it follows from (3.2) that

$$
\lambda(1-\kappa-\lambda)\left\|(I-U) y_{n}\right\|^{2} \leq\left(\left\|x_{n}-z\right\|^{2}-\left\|x_{n+1}-z\right\|^{2}\right) \rightarrow 0
$$

and

$$
\frac{(1-\tau)^{2}}{4} \frac{\left\|(I-T) A x_{n}\right\|^{4}}{\left\|A^{*}(I-T) A x_{n}\right\|^{2}} \leq\left(\left\|x_{n}-z\right\|^{2}-\left\|x_{n+1}-z\right\|^{2}\right) \rightarrow 0 .
$$

This implies that $\left\|(I-U) y_{n}\right\| \rightarrow 0$ and

$$
\frac{\left\|(I-T) A x_{n}\right\|^{2}}{\left\|A^{*}(I-T) A x_{n}\right\|} \rightarrow 0
$$

so that

$$
\begin{aligned}
\frac{1}{\|A\|}\left\|(I-T) A x_{n}\right\| & =\left\|(I-T) A x_{n}\right\| \frac{\left\|(I-T) A x_{n}\right\|}{\|A\|\left\|(I-T) A x_{n}\right\|} \\
& \leq\left\|(I-T) A x_{n}\right\| \frac{\left\|(I-T) A x_{n}\right\|}{\left\|A^{*}(I-T) A x_{n}\right\|} \\
& =\frac{\left\|(I-T) A x_{n}\right\|^{2}}{\left\|A^{*}(I-T) A x_{n}\right\|} \rightarrow 0,
\end{aligned}
$$

and also that

$$
\left\|x_{n}-y_{n}\right\|=\rho_{n}\left\|A^{*}(I-T) A x_{n}\right\|=\frac{1-\tau}{2} \frac{\left\|(I-T) A x_{n}\right\|^{2}}{\left\|A^{*}(I-T) A x_{n}\right\|} \rightarrow 0 .
$$

Having in mind that $\left\|(I-U) y_{n}\right\| \rightarrow 0$, we conclude that $\left\|(I-U) x_{n}\right\| \rightarrow 0$. Consequently (3.3) holds in both cases.

Finally, we show that $\left(x_{n}\right)$ converges weakly to $x^{*} \in S$. By Lemma 2.3, it remains to show that $\omega_{w}\left(x_{n}\right) \subseteq S$. To see this let $\hat{x} \in \omega_{w}\left(x_{n}\right)$ and let $\left\{x_{n_{k}}\right\}$ be a subsequence of $\left(x_{n}\right)$ converging weakly to $\hat{x}$. By noting that $\left\|(I-U) x_{n_{k}}\right\| \rightarrow 0$, we then make use of the demiclosedness of $I-U$ at zero to deduce that $\hat{x} \in \operatorname{Fix}(U)$; on the other hand, since, by weak continuity 
of $A, A x_{n_{k}}$ converges weakly to $A \hat{x}$ and $\left\|(I-T) A x_{n_{k}}\right\| \rightarrow 0$, this, together with the demiclosedness of $I-T$ at zero, yields $A \hat{x} \in \operatorname{Fix}(T)$. Altogether $\hat{x} \in S$, and therefore the proof is complete.

Remark 3.4 By Lemmas 2.3, we see that the weak limit $x^{*}$ of the sequence $\left(x_{n}\right)$ generated by Algorithm 3.1 coincides with the limit of the sequence $\left(P_{S} x_{n}\right)$, that is, $x^{*}=\lim _{n \rightarrow \infty} P_{S} x_{n}$. In fact, let $\hat{x}$ be the strong limit of the sequence $\left(P_{C} x_{n}\right)$. It follows from (2.3) that

$$
\left\langle x_{n}-P_{C} x_{n}, x^{*}-P_{C} x_{n}\right\rangle \leq 0 .
$$

Noting that $x_{n}-P_{C} x_{n} \rightarrow x^{*}-\hat{x}$ and $x^{*}-P_{C} x_{n} \rightarrow x^{*}-\hat{x}$, we obtain by sending $n \rightarrow \infty$ in (3.4)

$$
\left\|x^{*}-\hat{x}\right\|^{2}=\left\langle x^{*}-\hat{x}, x^{*}-\hat{x}\right\rangle \leq 0
$$

Hence, $x^{*}=\hat{x}$ and therefore $x^{*}=\lim _{n \rightarrow \infty} P_{S} x_{n}$.

\section{Some special cases}

\subsection{The case for quasi-nonexpansive operators}

Consider now the SCFP (1.2) under the following assumptions:

- $U: H \rightarrow H$ and $T: K \rightarrow K$ are both quasi-nonexpansive;

- both $I-U$ and $I-T$ are demiclosed at zero;

- it is consistent, i.e., its solution set, denoted by $S$, is nonempty.

Since every quasi-nonexpansive operator is clearly 0 -demicontractive, we can state the following result by using Algorithm 3.1.

Algorithm 4.1 Choose $0<\lambda<1$ and an initial guess $x_{0} \in H$ arbitrarily. Assume that the $n$th iterate $x_{n}$ has been constructed; then calculate the $(n+1)$ th iterate $x_{n+1}$ via the formula:

$$
x_{n+1}=U_{\lambda}\left(x_{n}-\rho_{n} A^{*}(I-T) A x_{n}\right),
$$

where the step $\rho_{n}$ is chosen in such a way that

$$
\rho_{n}:= \begin{cases}\frac{\left\|(I-T) A x_{n}\right\|^{2}}{2\left\|A^{*}(I-T) A x_{n}\right\|^{2}}, & A x_{n} \neq T\left(A x_{n}\right) \\ 0, & \text { otherwise. }\end{cases}
$$

Corollary 4.2 Let $\left(x_{n}\right)$ be the sequence generated by Algorithm 4.1. Then $\left(x_{n}\right)$ converges weakly to a solution $x^{*} \in S$.

\subsection{The case for directed operators}

Let us consider the SCFP (1.2) under the following assumptions:

- $U: H \rightarrow H$ and $T: K \rightarrow K$ are both directed;

- $I-U$ and $I-T$ are both demiclosed at zero;

- it is consistent, i.e., its solution set, denoted by $S$, is nonempty.

A simple calculation shows that every directed operator is -1-demicontractive. Thus we can state the following result by using Algorithm 3.1. 
Algorithm 4.3 Choose an initial guess $x_{0} \in H$ arbitrarily. Assume that the $n$th iterate $x_{n}$ has been constructed; then calculate the $(n+1)$ th iterate $x_{n+1}$ via the formula:

$$
x_{n+1}=U\left(x_{n}-\rho_{n} A^{*}(I-T) A x_{n}\right),
$$

where the step $\rho_{n}$ is chosen in such a way that

$$
\rho_{n}:= \begin{cases}\frac{\left\|(I-T) A x_{n}\right\|^{2}}{\left\|A^{*}(I-T) A x_{n}\right\|^{2}}, & A x_{n} \neq T\left(A x_{n}\right) \\ 0, & \text { otherwise. }\end{cases}
$$

Corollary 4.4 Let $\left(x_{n}\right)$ be the sequence generated by Algorithm 4.3. Then $\left(x_{n}\right)$ converges weakly to a solution $x^{*} \in S$.

Remark 4.5 Algorithm 4.3 covers the algorithm studied in [11] for solving the SFP. One can further apply the above result to the split variational inequality problem $[12,13]$ and the split common null point problem [14].

\section{Competing interests}

The authors declare that they have no competing interests.

\section{Authors' contributions}

All authors contributed equally to the writing of this paper. All authors read and approved the final manuscript.

\section{Acknowledgements}

This work is supported by the National Natural Science Foundation of China (Grant Nos. 11301253, 11271112).

Received: 30 September 2013 Accepted: 11 March 2014 Published: 25 Mar 2014

\section{References}

1. Censor, Y, Elfving, T: A multiprojection algorithms using Bregman projection in a product space. Numer. Algorithms 8 221-239 (1994)

2. Byrne, C: A unified treatment of some iterative algorithms in signal processing and image reconstruction. Inverse Probl. 18, 103-120 (2004)

3. Censor, Y, Bortfeld, T, Martin, B, Trofimov, A: A unified approach for inversion problems in intensity-modulated radiation therapy. Phys. Med. Biol. 51, 2353-2365 (2006)

4. Byrne, C: Iterative oblique projection onto convex sets and the split feasibility problem. Inverse Probl. 18, 441-453 (2002)

5. Censor, Y, Segal, A: The split common fixed point problem for directed operators. J. Convex Anal. 16, 587-600 (2009)

6. Moudafi, A: A note on the split common fixed-point problem for quasi-nonexpansive operators. Nonlinear Anal. 74(12), 4083-4087 (2011)

7. Moudafi, A: The split common fixed point problem for demicontractive mappings. Inverse Probl. 26, 055007 (2010)

8. Wang, F, Xu, HK: Cyclic algorithms for split feasibility problems in Hilbert spaces. Nonlinear Anal. 74, $4105-4111$ (2011)

9. Goebel, K, Kirk, WA: Topics in Metric Fixed Point Theory, vol. 28. Cambridge University Press, Cambridge (1990)

10. Bauschke, HH, Borwein, JM: On projection algorithms for solving convex feasibility problems. SIAM Rev. 38, 367-426 (1996)

11. López, G, Martín-Márquez, V, Wang, F, Xu, H-K: Solving the split feasibility problem without prior knowledge of matrix norms. Inverse Probl. 28, 085004 (2012)

12. Censor, Y, Gibali, A, Reich, S: Algorithms for the split variational inequality problem. Numer. Algorithms 59(2), 301-323 (2012)

13. Moudafi, A: Split monotone variational inclusions. J. Optim. Theory Appl. 150, 275-283 (2011)

14. Byrne, C, Censor, Y, Gibali, A, Reich, S: The split common null point problem. J. Nonlinear Convex Anal. 13, 759-775 (2011)

10.1186/1687-1812-2014-78

Cite this article as: Cui and Wang: Iterative methods for the split common fixed point problem in Hilbert spaces. Fixed Point Theory and Applications 2014, 2014:78 